\title{
Einführung - In einer Welt im Wandel, welche Positionierung für die Entwicklungszusammenarbeit?
}

Catherine Schümperli Younossian

\section{(2) OpenEdition}

Journals

Electronic version

URL: http://journals.openedition.org/sjep/98

DOI: $10.4000 /$ sjep.98

ISSN: 1663-9677

Publisher

Institut de hautes études internationales et du développement

\section{Printed version}

Date of publication: 1 avril 2007

Number of pages: XIII-XVII

ISBN: 978-2-88247-067-6

ISSN: $1660-5926$

Electronic reference

Catherine Schümperli Younossian, « Einführung - In einer Welt im Wandel, welche Positionierung für die Entwicklungszusammenarbeit? », Schweizerisches Jahrbuch für Entwicklungspolitik [Online], 26-1 | 2007, Online erschienen am: 20 November 2009, abgerufen am 24 September 2020. URL : http:// journals.openedition.org/sjep/98; DOI : https://doi.org/10.4000/sjep.98 


\section{Einführung}

\section{In einer Welt im Wandel, welche Positionierung für die Entwicklungszusammenarbeit?}

D AS JAHRBUCH hat zum Ziel, die wichtigsten Ereignisse betreffend die Entwicklungspolitik der Schweiz zu präsentieren. Jedoch geht es auch darum, diese Ereignisse im Rahmen der Veränderungen auf der internationalen Bühne zu sehen und die Folgen des Wandels für den Bereich der Entwicklungszusammenarbeit zu ermessen. Seit Anfang der 90er Jahre ist eine deutliche Weiterentwicklung des Kräfteverhältnisses zwischen Staaten festzustellen.

Osteuropäische Staaten, ein Übergangsprozess mit unterschiedlichem Tempo

Im Mai 2004 nahm die Europäische Union acht neue Staaten auf: die Baltischen Staaten (Litauen, Lettland, Estland) sowie fünf mitteleuropäische Länder (Polen, Tschechien, Slowakei, Ungarn und Slowenien). Im Januar 2007 kamen Rumänien und Bulgarien zur EU hinzu. Allerdings wird nur ein Drittel der 27 Transitionsländer von der Europäischen Kommission als stabile Rechtsstaaten mit einer wettbewerbsfähigen Volkswirtschaft betrachtet, die den EU-Beitritt rechtfertigt. Fünfzehn Jahre nach dem Fall der Berliner Mauer sind die politischen und wirtschaftlichen Reformen bei der Mehrheit dieser Länder, die sich sehr stark voneinander unterscheiden, noch nicht abgeschlossen. In Südosteuropa (Ex-Jugoslawien) und in vielen Ländern der Gemeinschaft Unabhängiger Staaten (GUS), insbesondere in Zentralasien (Usbekistan, Tadschikistan, Kirgistan) und im Südkaukasus (Georgien, Armenien und Aserbaidschan) bleibt die soziale, politische und wirtschaftliche Lage nach wie vor unsicher.

Die Schweiz, die ihre ersten Aktivitäten der Entwicklungszusammenarbeit in dieser Region 1990 durchführte, hat im November 2006 ein Gesetz über die künftige Tätigkeit der Zusammenarbeit erlassen. Diese Gesetzesgrundlage erlaubt es, dem Parlament weitere Kreditanträge zu unterbreiten. Das Gesetz wurde von den Eidgenössischen Räten mit grosser Mehrheit angenommen, jedoch von der Schweizerischen Volkspartei (SVP) angefochten, die das Referendum ergriff. Der Gesetzestext wurde schliesslich in der Volksabstimmung im Herbst 2006 vom Schweizer Volk mit knapper Mehrheit (53,4\% der Stimmen) angenommen. Das neue Gesetz betrifft zwei Hilfsformen der Schweiz: Zum einen ermöglicht es die Fortsetzung der Transitionshilfe (herkömmliche Entwicklungshilfe) für die Entwicklungsländer Osteuropas (Südosteuropa, Zentralasien und Südkaukasus). Zum anderen schafft es die rechtlichen Grundlagen für den Beitrag der Schweiz zur Verringerung des wirtschaftlichen und sozialen Gefälles in der erweiterten Europäischen Union.

Die Instrumente der Entwicklungszusammenarbeit wurden somit in der Schweiz sowie in anderen Ländern an die neuen Verhältnisse der Oststaaten angepasst. Der Entwicklungshilfeausschuss der OECD (DAC) überarbeitete im Jahr 2005 auch 
die Liste der Empfängerländer öffentlicher Hilfe. Die ursprüngliche Zweiteilung der Liste in Empfängerländer öffentlicher Entwicklungshilfe (APD) und Empfängerländer öffentlicher Hilfe (AP) wurde abgeschafft; die Liste besteht nur noch aus einem Teil. Weissrussland und die Ukraine wurden in die neue Liste der Empfängerländer öffentlicher Entwicklungshilfe aufgenommen. Die übrigen Länder, die in der Vergangenheit öffentliche Hilfe erhielten, (Bulgarien, Tschechische Republik, Estland, Ungarn, Lettland, Litauen, Polen, Rumänien, Russland und Slowakei) erscheinen nicht mehr in den DAC-Statistiken. Mit dieser statistischen Vereinfachung wird unbemerkt ein neues Bild dieser Staaten vermittelt.

Neue internationale Schwergewichte: China und Indien

Seit kurzem steht das Aufkommen einiger „Länder des Südens“, die allgemein mit dem Begriff „BRIC““ (Brasilien, Russland, Indien, China) bezeichnet werden, im Rampenlicht. Falls ihr derzeitiges Wachstumstempo anhält, dürfte das kumulierte Bruttoinlandsprodukt dieser Länder in rund dreissig Jahren jenes der heutigen sechs grössten Volkswirtschaften (Vereinigte Staaten, Japan, Deutschland, Frankreich, Grossbritannien und Italien) übertreffen. Die Wirtschaft der BRICLänder bleibt zwar instabil und ihr Markt volatil, doch nimmt das politische Gewicht dieser Staaten von Jahr zu Jahr zu. 2006 hat der Bundesrat spezifische Strategien für die Wirtschaftspolitik der Schweiz gegenüber den BRIC-Ländern verabschiedet. Das Einleitungskapitel des Berichts zur Aussenwirtschaftspolitik $2006^{1}$ befasst sich mit der weltwirtschaftlichen Bedeutung der BRIC-Länder und mit den Implikationen für die Schweiz.

Das Aufkommen dieser neuen Wirtschaftsmächte hat auch Auswirkungen auf andere Entwicklungsregionen. Zur Veranschaulichung der Problematik versuchten Forscher der OECD, das Gewicht und den Einfluss Chinas und Indiens auf dem afrikanischen Kontinent zu messen: China und Indien würden wegen ihrer Grösse, wegen des sensationellen Wachstumstempos, des enormen Verbrauchs an natürlichen Ressourcen und der Zunahme ihrer wirtschaftlichen und politischen Macht die Weltwirtschaft unweigerlich umgestalten und deren Spielregeln beeinflussen ${ }^{2}$. Die Studie zieht das Fazit, dass die afrikanischen Länder den wirtschaftlichen Sprung Chinas und Indiens nicht nur passiv miterleben, sondern auch aktiv daran teilnehmen. Die Wirtschaftsmacht der beiden asiatischen Riesen beeinflusst bereits heute die Wachstumsmuster in Afrika, besonders derjenigen Länder, die Erdöl und Grundstoffe exportieren. So drängt sich die Feststellung auf, dass bestimmte afrikanische Länder sich heute von ihren traditionellen OECD-Partnern abwenden und den beiden neuen Wirtschaftsmächten zuwenden. Das Handelsvolumen ist ein anschauliches Beispiel dafür: Der Handel zwischen China und dem afrikanischen Kontinent nahm zwischen 1980 und 2005 um das 50fache zu; er stieg von 12,39 Milliarden Dollar im Jahr 2002 auf 40 Milliarden im Jahr 2005 und 50 Milliarden im Jahr $2006^{3}$ an.

Das Forum China-Afrika, das im November 2006 in Peking tagte, hat diesen Bündniswechsel noch bestätigt. Nur wenige Medien berichteten über die Veran-

1 Bundesrat, Bericht zur Aussenwirtschaftspolitik 2006 vom 10 Januar 2007 (BB1 2007 897).

2 A. Golstein, N. Pinaud, H. Reisen und X. Chen, L'essor de la Chine et de l'Inde. Quels enjeux pour l'Afrique?, Etudes du Centre de développement, Paris, OCDE, 2006.

3 „Comment la Chine a conquis les Africains“, L'Hebdo, 25. Januar 2007. 
staltung, obwohl 48 Staatschefs (von 53 afrikanischen Ländern) dem Appell des Präsidenten der Volksrepublik China, Hu Jintao, Folge leisteten. Wie ein Artikel der Zeitschrift L'Hebdo festhält, sind diese Beziehungen nicht nur rein kommerzieller Art: Peking beabsichtige, zur Entwicklung des Schwarzen Kontinents beizutragen, und entsende Tausende von Arbeitnehmern und Entwicklungshelfern in allen Sektoren: Bauwesen (Strassen, Eisenbahn, Amtsbauten, Hotelanlagen, Mietsgebäude), Gesundheitswesen (Eröffnung von Krankenhäusern und Krankenstationen, Versorgung mit Generika, chinesische Medizin, Impfungen, Prävention von Aids und Malaria), kultureller Austausch (Tausende Stipendien für ein Studium in China, chinesische Kulturwochen in Afrika). ${ }^{4}$

Die neue Situation wirft zahlreiche Fragen auf: Wie wirkt sich die starke und rasch wachsende Nachfrage nach natürlichen Ressourcen (Rohstoffe und Erdöl) auf die Umwelt aus? Wie beeinflussen die massiven Importe von Produkten Made in China die lokalen Märkte? Wie lassen sich die Asymmetrie des neuen Handelsaustauschs und neue Abhängigkeiten bewältigen? Auf welche Weise werden die ehemaligen Kolonialmetropolen ihr Angebot und ihre Haltung gegenüber den afrikanischen Ländern überprüfen? Wie wird garantiert, dass die neuen Konzepte und Praktiken der Entwicklungszusammenarbeit, die im Rahmen des DAC eingeführt wurden (Erklärung von Rom über die Harmonisierung der Hilfe, Erklärung von Paris über die Wirksamkeit der Hilfe), in diesem neuen Kontext ihre Gültigkeit behalten können? Dies sind einige der wichtigsten Fragen, vor denen die traditionellen Akteure der Entwicklungszusammenarbeit stehen.

Auf einer anderen Ebene geht es jetzt nachstehend um die Probleme der schweizerischen Entwicklungspolitik.

Schweizerische Entwicklungszusammenarbeit: politische Termine im Jahr 2007

2007 ist für die Entwicklungszusammenarbeit der Schweiz ein entscheidendes Jahr. Die Entwicklungszusammenarbeit geniesst zwar einen guten Ruf und besitzt in der Schweizer Bevölkerung grossen Rückhalt, wurde aber in den letzten Jahren von den konservativen Kreisen heftig unter Beschuss genommen. Diese Kreise sind darauf aus, der Entwicklungszusammenarbeit „,die Flügel zu stutzen“, indem sie versuchen, die Finanzierung zu reduzieren und mit technischen Argumenten die Funktionsweise zu diskreditieren.

2007 muss das Parlament in einem angespannten politischen Klima den Finanzrahmen für die öffentliche Entwicklungshilfe (APD) der nächsten vier bis fünf Jahre festlegen. Im Laufe des Jahres 2007 müssen nämlich fünf neue Rahmenkredite für folgende Bereiche genehmigt werden: humanitäre Hilfe, Globaler Umweltfonds (GEF), Zusammenarbeit in Osteuropa, wirtschafts- und handelspolitische Massnahmen (SECO), technische Zusammenarbeit und Finanzhilfe für die Länder des Südens (DEZA). Zu dieser finanziellen Dimension kommen noch die politischen Auswirkungen hinzu, die von dem im Dezember 2006 veröffentlichten Bericht der Geschäftsprüfungskommission des Ständerates unweigerlich aufgeworfen werden 5 .

4 Ibid.

5 Geschäftsprüfungskommission des Ständerates, Kohärenz und strategische Führung der Aktivitäten der DEZA, 8. Dezember 2006. 
Die parlamentarischen Beratungen im Jahr 2007 über die Zuweisung der Beträge für die Entwicklungszusammenarbeit sind aus der Warte der nationalen Politik wichtig. Sie werden Hinweise auf die unterschiedlichen Positionen zu diesem Kapitel der Aussenpolitik der Schweiz und Aufschluss über den Willen zum schweizerischen Engagement in der internationalen Zusammenarbeit geben. Die Schweiz bringt 0,44 Prozent ihres Bruttonationaleinkommens (BNE) für die öffentliche Entwicklungshilfe auf und liegt damit unter den 22 DAC-Ländern an elfter Stelle. Die Verbesserung dieses Prozentsatzes geht zwar auf eine neue Verbuchung des Aggregats der öffentlichen Entwickungshilfe zurück, jedoch ist auch zu erwähnen, dass viele Länder diese vom DAC festgelegte Definition frei auslegen.

Das finanzielle Engagement eines Staates entgeht der Aufmerksamkeit der internationalen Gemeinschaft nicht. Deshalb steht hier das „Image“ der Schweiz auf dem Spiel. Die Industrieländer, die den Löwenanteil der Finanzierung der öffentlichen Entwickungshilfe bestreiten, legen Wert auf eine gerechte Aufteilung. Gerechtigkeit setzt jedoch voraus, dass die reichsten Länder sich stärker als die anderen engagieren. In dieser Hinsicht hat die Schweiz angesichts ihres Lebensstandards offensichtlich noch einige Anstrengungen zu leisten.

\section{Vertiefung der Thematik der Entwicklungsfinanzierung: Dossier des Jahrbuchs 2007}

Die Frage der Entwicklungsfinanzierung steht im Mittelpunkt der Diskussionen über die internationale Zusammenarbeit. Das Konzept wird indes zu häufig einschränkend als öffentliche Entwicklungshilfe (APD) ausgelegt - eine Teilsicht, da diese Hilfsflüsse im Vergleich zu anderen internationalen Finanzflüssen, die sich ebenfalls auf die Entwicklung auswirken, zum Beispiel Auslandsinvestitionen, oft lächerlich gering sind. Die Beträge öffentlicher Entwicklungshilfe (108 Milliarden Dollar im Jahr 2005) müssen auch gegenüber den Geldüberweisungen der Migranten in die Heimat relativiert werden, die gemäss der Internationalen Organisation für Migration (IMO) 2005 insgesamt rund 167 Milliarden Dollar betrugen.

Ein Erfolg der Konferenz von Monterrey im Jahr 2002 bestand darin, dass ein globaler Aktionsplan erstellt wurde, der die wichtigsten Finanzierungsquellen für die Entwicklung offenlegte. Die Konferenz stellte fest - ohne die Bedeutung der öffentlichen Entwicklungshilfe herunterzuspielen -, dass auch die massivste Aussenhilfe niemals die grundlegenden Reformen ersetzen kann, die die Volkswirtschaften brauchen. Als wichtigster Durchbruch der Konferenz von Monterrey gilt, dass die Finanzierung der Entwicklung mit der Identifizierung von sechs komplementären Handlungsbereichen nun breiter gesehen wird. Die öffentliche Entwicklungshilfe, die weiterhin einen Tragpfeiler der Entwicklungszusammenarbeit bildet, wird ergänzt durch die Mobilisierung nationaler Ressourcen, innovative Finanzierungsmechanismen (z.B. neue Abgaben auf Kerosin, Flugtickets oder Waffen), Auslandsinvestitionen, die Schuldenfrage (und mithin die Entschuldung) und den internationalen Handel. So wird fünf Jahre nach der Konferenz von Monterrey das nächste Dossier des Jahrbuchs, das im Herbst 2007 erscheint, die Initiativen und Fortschritte in diesem Bereich umreissen. 
Ein herzlicher Dank des Redaktionskomitees geht an die Autorinnen und Autoren der im vorliegenden Band Schweizerisches Jahrbuch für Entwicklungspolitik veröffentlichten Texte sowie an die zahlreichen Spezialisten bei den Bundesbehörden, den Entwicklungs-NRO und Akademikerkreisen, welche durch ihre kritische Überprüfung und ihre wertvollen Ratschläge unserer Publikation zu ihrer Seriosität verholfen haben. Unser Dank geht auch an all jene, die zur Gestaltung, Realisierung und Übersetzung des Werks beigetragen haben. Auf ihrer Kompetenz und ihrem Engagement beruht die Zuverlässigkeit dieses Nachschlagewerks.

Catherine Schümperli Younossian

Redaktionsleiterin 